\title{
Sequentially Deposited Compact and Pinhole-Free Perovskite Layers via Adjusting the Permittivity of the Conversion Solution
}

https://doi.org/10.1515/zna-2019-0141

Received April 26, 2019; accepted June 12, 2019; previously

published online July 2, 2019

\begin{abstract}
The preparation of compact and pinhole-free absorber layers is a major step towards device reproducibility and high performance for planar organicinorganic perovskite solar cells. It is well known that the sequential deposition method exhibits some advantages over the common one-pot synthesis in terms of controlling the surface coverage. However, it still miscarries to produce pinhole-free layers from solution, mainly due to the occurrence of dissolution and recrystallisation processes. We show that by a careful choice of the permittivity of the alcoholic solvent in the conversion step the surface morphology can be finely modified, thereby yielding pinhole-free and compact absorber films comparable to that from vapour-assisted solution techniques. It is observed that the permittivity controls the intensity of the Ostwald ripening effect and that a low value of the former enables an in situ intercalation of precursor materials into the lead halide framework. We successfully prepared smooth and mirror-like perovskite surfaces that demonstrate enhanced optoelectronic properties and photovoltaic performance compared to films from the native two-step deposition in isopropanol. This strategy provides a facile approach for obtaining high-quality layers in the planar architecture by simple solution processing.
\end{abstract}

\footnotetext{
*Corresponding author: Michael Wark, Chemical Technology 1, Photocatalysis and Sustainable Feedstock Utilization, Institute of Chemistry, Faculty of Mathematics and Natural Sciences, Carl von Ossietzky University Oldenburg, Carl-von-Ossietzky Str. 9-11, 26129 Oldenburg, Germany, E-mail: michael.wark@uni-oldenburg.de. https://orcid.org/0000-0002-8725-0103

Markus Becker: Chemical Technology 1, Photocatalysis and Sustainable Feedstock Utilization, Institute of Chemistry, Faculty of Mathematics and Natural Sciences, Carl von Ossietzky University Oldenburg, Carl-von-Ossietzky Str. 9-11, 26129 Oldenburg, Germany; and Fraunhofer Institute for Manufacturing Technology and Advanced Materials IFAM, Wiener Strasse 12, 28359 Bremen, Germany, E-mail: markus.becker@ifam.fraunhofer.de. https://orcid.org/0000-0002-7000-0802
}

Keywords: Carrier Lifetimes; Dissolution-Recrystallisation; Perovskite Solar Cells; Sequential Deposition; Solvent Permittivity.

Dedicated to: Prof. Dr. J. Parisi on occasion of his retirement.

\section{Introduction}

Hybrid organic-inorganic perovskites have recently attracted tremendous attention as absorber materials in the next generation of photovoltaics, which can be mainly ascribed to their phenomenal increase in power conversion efficiencies (PCE) [1-7]. In less than 10 years, the performance has been raised from initially $3.8 \%$ [8] to a certified record value of $24.2 \%$ [9]. These high efficiencies combined with the ease of solution-based processability as well as the low cost of the precursor materials render them superior compared to other thin film technologies, e.g. dye sensitised solar cells, organic photovoltaics, or quantum dot sensitised solar cells [10]. It also indicates a possible economical way to challenge crystalline silicon [11]. Excellent optoelectronic properties, such as low exciton-binding energies [12], strong optical absorption [13], and the extraordinary long charge carrier diffusion lengths [14], attract immense interest for future commercialisation. The latter fact can also be attributed to long-term pricing concerns in certain systems like cadmium telluride or copper indium gallium diselenide, as the availability and costs of their precursor materials may limit the commercial success [15]. However, some concerns have arisen due to the toxicity of lead as well as moisture-dependent instability, both of which may slow down commercial implementation [16, 17]. Besides the possibility of optimising the interfaces between the absorber material and the selective contacts for promoting improved charge carrier separations [18-22], the morphology of the absorber surface is of prime importance considering the photovoltaic efficiency in a perovskite solar cell $[6,23,24]$. Particularly in the planar heterojunction configuration, latest advancements in the multiple fabrication routes of the absorber layer have enabled a rapid performance increase. However, these results have 
also discovered the decisive role of a constant thickness and compact morphology [25].

During the beginning of perovskite solar cell research, the single-step deposition of mixed precursor solutions containing lead iodide $\left(\mathrm{PbI}_{2}\right)$ and methyl ammonium iodide $\left(\mathrm{CH}_{3} \mathrm{NH}_{3} \mathrm{I}\right.$ or MAI) from common solvents such as $N, N$-dimethylformamide (DMF), dimethylsulfoxide, or $\gamma$ butyrolactone has been conducted [26, 27]. As the resulting films typically exhibited poor surface coverages with uncovered voids and/or pinholes at the early stages, both of which led to substantial leakage currents and negatively affected charge carrier transports, the sequential deposition has been developed [28, 29]. In this method, a $\mathrm{PbI}_{2}$ precursor layer is deposited via spin-coating from a solution in DMF and afterwards immersed into an isopropanolic (IPA) solution of MAI. Due to the templating behaviour of $\mathrm{PbI}_{2}$, a better morphology control can be achieved that allows the preparation of thin films with high surface coverages and superior device reproducibility. However, when prepared from the common IPA solvent, the $\mathrm{PbI}_{2}$ precursor crystals partially dissolve and recrystallise into irregular cuboid perovskite crystallites (Ostwald ripening process) [30]. This, in turn, has detrimental effects on the photovoltaic performance, due to the presence of a nonuniform crystallite size distribution and large number of grain boundaries. Recently, Hsieh et al. [31] have elucidated the evolution of methyl ammonium lead triiodide ( $\left.\mathrm{MAPbI}_{3}\right)$ surface morphologies along with the immersion times of $\mathrm{PbI}_{2}$ into MAI/IPA conversion solutions and observed two dominating time periods. In the first period, the interfacial reaction of $\mathrm{PbI}_{2}$ and MAI is perking up, whereas in the second timeframe the dissolution-recrystallisation process is dominating. It is stated that the optimal condition for the preparation of $\mathrm{MAPbI}_{3}$ layers is at the point between these two timescales, meaning that the conversion to the perovskite phase should be complete, but the crystallites do not suffer too much from dissolution. Additionally, Yang et al. [30] have found via the investigation of suspended $\mathrm{PbI}_{2}$ microcrystals that short-chained polar solvents favour the dissolution-recrystallisation behaviour, leading to larger grain sizes, whereas long chained nonpolar solvents are unfavourable for the diffusion of the solute. Although it is generally assumed that larger crystallites result in enhanced charge carrier mobilities, high solar cell performances can only be achieved with the highest surface coverages, requiring suppressed recombination between the $n$ - and $p$-type selective contacts at the pinholes and/or uncovered voids [6, 32, 33].

In this study, we examine the sequential deposition of planar $\mathrm{MAPbI}_{3}$ films from MAI solutions prepared from the low permittivity solvent pentan-1-ol. It is observed that the
$\mathrm{PbI}_{2}$ dissolution can be significantly suppressed compared to the use of conventional IPA and that the effective in situ intercalation of MAI into the $\mathrm{PbI}_{2}$ framework results in extremely dense perovskite films with mirror-like appearance. Films fabricated from pure pentan-1-ol demonstrate stronger optical absorption and enhanced charge carrier lifetimes. Furthermore, the absorber layers show surface morphologies comparable to films prepared from vapourassisted solution methods, and the PCE can be enhanced by approximately $60 \%$. It is expected that the strategy of permittivity adjustment can lead to significant improvement in the native solution-based two-step preparation of absorber layers in planar heterojunction perovskite solar cells.

\section{Experimental Section}

\subsection{Material Synthesis}

The $\mathrm{PbI}_{2}$ crystals were prepared as follows: typically, $10.78 \mathrm{~g}$ of lead nitrate $\left[\mathrm{Pb}\left(\mathrm{NO}_{3}\right)_{2},>99 \%\right.$; Sigma-Aldrich, St. Louis, MO, USA] and $10.80 \mathrm{~g}$ of potassium iodide (KI, $>99 \%$; Sigma-Aldrich) were separately dissolved in $50 \mathrm{~mL}$ of deionised water. Then, the KI solution was slowly added to the $\mathrm{Pb}\left(\mathrm{NO}_{3}\right)_{2}$ solution under vigorous stirring, whereby a yellow suspension was obtained. The suspension was filtrated, and the precipitate was rinsed with deionised water several times. Finally, the yellow solid was dried in a vacuum furnace at $70{ }^{\circ} \mathrm{C}$ for $24 \mathrm{~h}$ and subsequently stored in Argon.

$\mathrm{CH}_{3} \mathrm{NH}_{3} \mathrm{I}$ was synthesised by reacting equimolar amounts of methyl amine $\left(\mathrm{CH}_{3} \mathrm{NH}_{2} 40\right.$ wt.\% aqueous solution; Alfa Aesar, Ward Hill, MA, USA) and hydroiodic acid (HI, 57 wt.\% aqueous solution; Sigma-Aldrich). Usually, $5 \mathrm{~mL}$ of methyl amine were diluted with $5 \mathrm{~mL}$ of ethanol (EtOH absolute, $>99.5 \%$; Fisher Scientific, Waltham, $\mathrm{MA}, \mathrm{USA}$ ) and stirred at $0{ }^{\circ} \mathrm{C}$ in a $50 \mathrm{~mL}$ round-bottom flask; $7.65 \mathrm{~mL}$ of the HI solution were slowly added, and the mixture was stirred for $2 \mathrm{~h}$ at $0{ }^{\circ} \mathrm{C}$. After warming to room temperature, the solution was allowed to stir for another $2 \mathrm{~h}$. A brown precipitate was obtained by evaporating the solvent at $50^{\circ} \mathrm{C}$ and 110 mbar for $2 \mathrm{~h}$ and subsequently at 30 mbar for another hour. The solid was recrystallised in ethanol and washed with diethyl ether three times. Finally, the colourless solid was dried in a vacuum furnace at $60^{\circ} \mathrm{C}$ for $24 \mathrm{~h}$.

\subsection{Device Fabrication}

Fluorine doped tin oxide (FTO) glass substrates (NSG Tec, $7 \mathrm{Ohm} / \mathrm{cm}^{2}$; Pilkington, St. Helens, UK) were cleaned sequentially for $10 \mathrm{~min}$ in a sonicator with deionised water, acetone, and IPA, respectively. The $\mathrm{PbI}_{2}$ precursor films were prepared by spin-coating $\mathrm{PbI}_{2}$ in DMF (>99.8 \%; Sigma-Aldrich) solutions with concentrations of 0.5 or $0.75 \mathrm{M}$ at a spin speed of $4000 \mathrm{rpm}$ for $25 \mathrm{~s}$. Spin-coating was performed in a static dispense way, meaning that the solution was dropped onto the surface before the spin-coater was started. The substrate and solution temperatures were held at $65^{\circ} \mathrm{C}$ directly before deposition. After spin-coating, the films were dried at $65^{\circ} \mathrm{C}$ for $10 \mathrm{~min}$ and subsequently cooled to the desired temperature for the conversion $\left(25^{\circ} \mathrm{C}\right.$ or $40^{\circ} \mathrm{C}$, respectively). The substrates were subsequently 
immersed into a solution of MAI in pentan-1-ol ( $>99 \%$, Merck, Darmstadt, Germany) with concentrations of $7.5,10.0$, or $12.5 \mathrm{mg} / \mathrm{mL}$ for time intervals of 2 and $6 \mathrm{~min}$, respectively. The temperature of the immersion solution was constantly held within $\pm 2{ }^{\circ} \mathrm{C}$ during the dipping timeframe. A subsequent immersion into the conversion solution is industrially attractive due to being an easily applicable and inexpensive approach. Furthermore, a sophisticated control of the MAI concentration and solution temperature allows the fabrication of firmly crystallised and highly reproducible absorber layers. Excess MAI/pentan-1-ol solution was removed by spin-coating the substrates at $4000 \mathrm{rpm}$ for $20 \mathrm{~s}$. Finally, the films were baked on a hot plate at $100{ }^{\circ} \mathrm{C}$ for $5 \mathrm{~min}$. All device fabrication steps were performed in dry ambient atmosphere at a temperature of $25^{\circ} \mathrm{C}$ and a relative humidity of $20-25 \%$.

For the preparation of photovoltaic devices, the FTO substrates were covered with a compact $\mathrm{TiO}_{2}$ hole-blocking layer via spin-coating a medium acidic solution of titanium isopropoxide (97\%; Sigma-Aldrich) in EtOH absolute at $6000 \mathrm{rpm}$ for $30 \mathrm{~s}$. Afterwards, the substrates were calcined in air for $30 \mathrm{~min}$ at $500{ }^{\circ} \mathrm{C}$, in order to obtain the anatase phase of $\mathrm{TiO}_{2}$. The photoactive layers were prepared as described above. A hole transport layer was prepared by spin-coating a solution of 2,2',7,7'-Tetrakis-( $N, N$-di4-methoxyphenylamino)-9,9'-spirobifluorene (Spiro-OMeTAD, $99 \%$, Sigma Aldrich) with a concentration of $100 \mathrm{mg} / \mathrm{mL}$ in chlorobenzene at $1500 \mathrm{rpm}$ for $45 \mathrm{~s}$, with additives of lithium-bis (trifluoromethanesulfonyl) imide salt $(5 \mathrm{mg} / \mathrm{mL}, 99.95 \%$, Sigma Aldrich) and 4-tert-butylpyridine (4-tBP, $10 \mu \mathrm{L} / \mathrm{mL}, 98 \%$, Sigma Aldrich). The devices were stored in a desiccator for $24 \mathrm{~h}$ in order to induce doping via oxygen molecules and to enhance the hole conductivity. Afterwards, $40 \mathrm{~nm}$ Au back electrodes were deposited by sputtering with a shadow mask defining an active area of $0.16 \mathrm{~cm}^{2}$.

\subsection{Characterisation}

Film morphologies were characterised by scanning electron microscopy (SEM; Helios Nanolab 600i, 5 kV, 0.17 nA; FEI Fisher Scientific), whereas the surface roughness was recorded with a Dektak $6 \mathrm{M}$ stylus profilometer (Veeco Instruments, Plainview, NY, USA). The optical transmission was measured with a Cary Varian 4000 spectrophotometer (former Varian Inc., now Agilent Technologies, Santa Clara, CA, USA) in the range of 400-900 nm. All photoluminescence (PL) measurements were recorded with a nitrogen cooled photomultiplier $\left(-80{ }^{\circ} \mathrm{C}\right.$, R5509; Hamamatsu Photonics, Hamamatsu, Japan) and a time-correlated single-photon counter (SPC 130; Becker \& Hickl GmbH, Berlin, Germany) in air. Due to these measurements, no deterioration of the films was observed. Excitation was provided at $650 \mathrm{~nm}$ via a dye laser employing 4-(dicyanomethylene)-2-methyl6-(4-dimethylaminostyryl)-4H-pyran (DCM) as dye with a pulse width of $7 \mathrm{ps}$, a pulse energy of $0.25 \mathrm{~nJ}$, a repetition rate of $2 \mathrm{MHz}$, and a spot size of $0.4 \mathrm{~mm}^{2}$. The time-resolved PL signals were monitored at the spectral peak $(\sim 780 \mathrm{~nm})$, and the excitation wavelength of $715 \mathrm{~nm}$ was adjusted with a cutoff filter. Crystallographic properties of the films were investigated by X-ray diffraction (XRD, $\mathrm{Cu} \mathrm{K \alpha}$ radiation, $40 \mathrm{kV}$; Panalytical Empyrean Almelo, The Netherlands). Current-density-voltage $(\mathrm{J}-\mathrm{V})$ measurements were conducted with a Xenon lamp-based solar simulator (model 69911, 200 W; LOT Oriel, Darmstadt, Germany) with a simulated AM 1.5 irradiation and an intensity of $100 \mathrm{~mW} / \mathrm{cm}^{2}$ (calibrated with a thermopile CA2; Kipp \& Zonen, Delft, The Netherlands).

\section{Results and Discussion}

The two-step synthesis method holds certain advantages over the one-step preparation in terms of controlling the crystallisation and morphology of perovskite thin films [34]. Therefore, in this study, the sequential deposition is adopted to fabricate planar absorber layers, where a $\mathrm{PbI}_{2}$ precursor film is spin-coated on top of the flat substrate and subsequently immersed into an alcoholic solution of MAI (see Experimental Section for details). Recently, the drawback of intrinsic dissolution-recrystallisation in the two-step method has been tackled by investigating solvents with different molecular geometries. It has been observed that branched structures, e.g. tert-butanol, can facilitate the in situ intercalation of MAI into the $\mathrm{PbI}_{2}$ framework, benefiting the formation of smooth and nearly pinhole-free perovskite films [30]. This has stimulated us to expand the search for potential solvents, which suppress the Ostwald ripening, via considering their static permittivity $(\varepsilon)$. Pure molecular geometries or dipole moments represent properties acting in the microscopic scale, whereas the permittivity as a macroscopic feature also includes the polarisability effects $[35,36]$. The static relative permittivity is expressed as the ratio of the absolute permittivity of the solvent to that of the vacuum. It is chosen to be the representative polarity parameter in this study as our former investigation has revealed a strong correlation to the average grain size as well as intensity of Ostwald ripening [37]. As ambient water vapour might influence the effective permittivity of the conversion solution, all samples are prepared under similar dry atmospheric conditions (i.e. relative humidity between 20 and $25 \%$ at a temperature of $25{ }^{\circ} \mathrm{C}$ ). Pentan-1-ol has been found to exhibit a lower permittivity compared to IPA (13.9 vs. 18.3 As/Vm for pentan-1-ol and IPA, respectively) but a similar dipole moment (1.70 vs. $1.66 \mathrm{D}$ for pentan-1-ol and IPA, respectively). Therefore, pentan-1-ol is expected to meet three important features: a significantly suppressed dissolution-recrystallisation of $\mathrm{PbI}_{2}$, a high phase conversion ratio, and the ability of sufficient MAI dissolution (above $\sim 10 \mathrm{mg} / \mathrm{mL}$ ).

We explored the surface morphology of $\mathrm{MAPbI}_{3}$ absorber layers processed from various MAI/pentan-1-ol mixtures via SEM imaging. For that, the $\mathrm{PbI}_{2}$ precursor films were immersed into conversion solutions with alternating concentrations and temperatures as well as for different durations. The results demonstrate that the MAI concentration, as well as the $\mathrm{PbI}_{2}$ precursor layer thickness, have a critical impact on the perovskite morphology, whereas the solution temperature and the immersion time seem to play minor roles. The initial $\mathrm{PbI}_{2}$ layer 


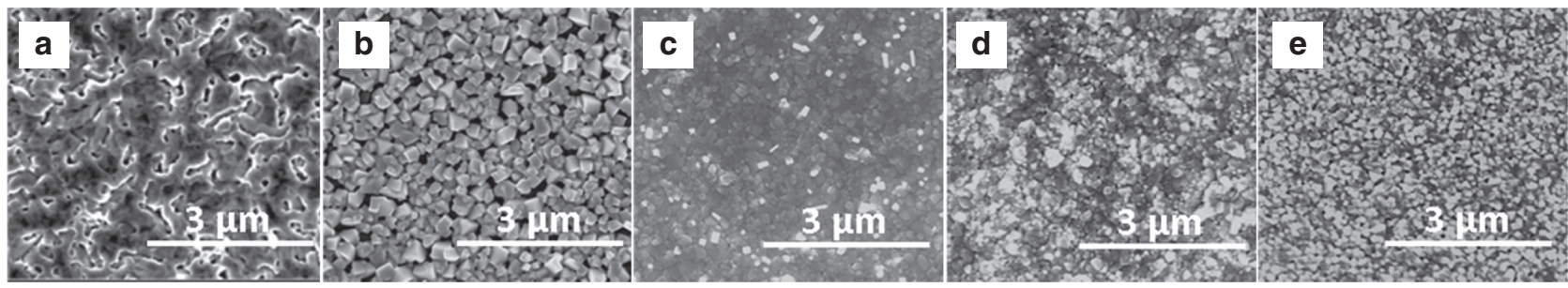

Figure 1: Top-view SEM images of (a) the $\mathrm{Pbl}_{2}$ precursor layer and (b-e) $\mathrm{MAPbl}_{3}$ perovskite films prepared by the sequential deposition method. A $10 \mathrm{mg} / \mathrm{mL}$ pristine MAI/IPA conversion solution was used for (b), whereas MAI/pentan-1-ol mixtures with concentrations of (c) 12.5 , (d) 10.0 , and (e) $7.5 \mathrm{mg} / \mathrm{mL}$ were applied to investigate the effect of the low permittivity on the crystal growth.

is shown in Figure 1a, revealing distinct and spatially repeated sheets that are oriented flat over the compact substrate. Commonly, IPA is applied as the standard solvent for MAI dissolution [38-43], but as can be seen from Figure 1b, significant Ostwald ripening leads to the typically observed rough and nonuniform surface with considerable amounts of uncovered voids and pinholes. Different MAI/IPA concentrations were tested in the conversion reaction; however, a strong dissolutionrecrystallisation can always be observed, and larger grains are typically obtained by lower concentrations (Fig. 2). On the other hand, an extremely smooth and pinhole-free $\mathrm{MAPbI}_{3}$ film is obtained by immersing the $\mathrm{PbI}_{2}$ precursor into a highly concentrated MAI/pentan-1-ol solution $(\mathrm{c}[\mathrm{MAI}]=12.5 \mathrm{mg} / \mathrm{mL})$, demonstrating a surface coverage of $100 \%$ (Fig. 1c). With increasing $\mathrm{PbI}_{2}$ layer thickness, the absorber morphology becomes somewhat inhomogeneous due to the development of height inconsistencies (Fig. S1 in the Supporting information). The small and dense perovskite grains in these films contribute to a superior homogeneity and are thus expected to improve the reproducibility of photovoltaic devices.

Most intriguingly, the as-prepared films exhibit a mirror-like appearance, which even reflects the background characters in the digital image (Fig. 3). With lower concentrations of $\mathrm{MAI} /$ pentan-1-ol, the perovskite surface seems to follow a dissolution-recrystallisation behaviour. This is indicated by the nonuniform crystallite size distribution and the rather isolated grains (Fig. 1e), which are comparable to that from IPA-based films. The anisotropic crystal growth results in rather adverse morphological properties, revealing several holes with diameters between roughly 10 and $100 \mathrm{~nm}$. A moderate MAI concentration $(10 \mathrm{mg} / \mathrm{mL})$ gives rise to surface characteristics at the transition point (Fig. 1d). These films represent itself as a combination of in situ-grown continuous, pinhole-free layers and rough dissolved-recrystallised surfaces typically observed from pristine IPA.

When higher temperatures are applied during the conversion reaction (i.e. $T=40^{\circ} \mathrm{C}$ ), the trends of morphology variations remain more or less the same; however, the appearance of dissolution-recrystallisation is already observed at median MAI concentrations (Fig. S1 in the Supporting information). This observation indicates that the intensity of Ostwald ripening for both, $\mathrm{PbI}_{2}$ and $\mathrm{MAPbI}_{3}$, is promoted by the higher thermal energy of the solvent molecules.

Surface textures were determined by measuring the arithmetical mean deviation of the assessed profile (denoted as Ra value). The average roughness of the $\mathrm{PbI}_{2}$ precursor layer is found to be $7.3 \pm 0.7 \mathrm{~nm}$. After the conversion to the $\mathrm{MAPbI}_{3}$ phase in the conventional IPA solvent, this value lowers to $4.9 \pm 0.8 \mathrm{~nm}$. However, by using the highly concentrated MAI/pentan-1-ol solution, the value can be further decreased to $4.2 \pm 0.5 \mathrm{~nm}$ accompanied by an improved reproducibility. It is expected that
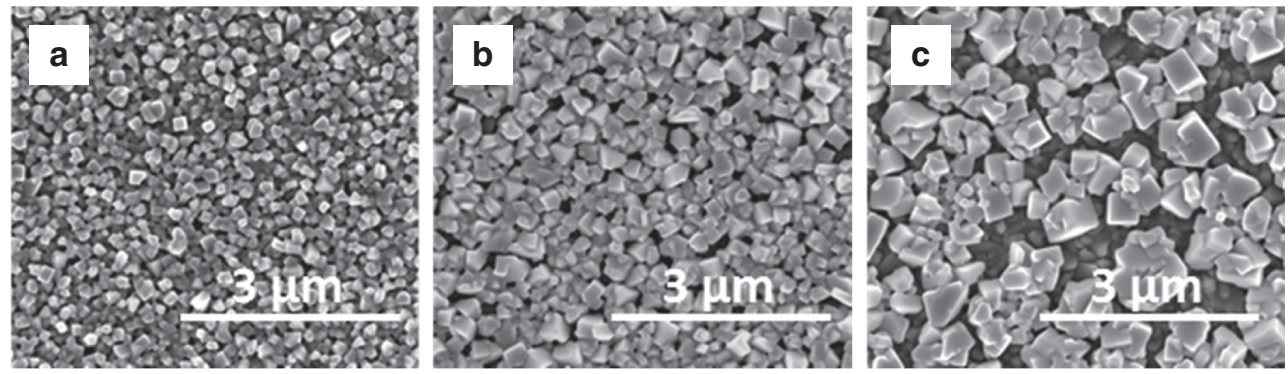

Figure 2: Top-view SEM images of $\mathrm{MAPbl}_{3}$ absorber layers prepared from MAI/IPA solutions with concentrations of (a) 12.5, (b) 10.0, and (c) $7.5 \mathrm{mg} / \mathrm{mL}$, respectively. 


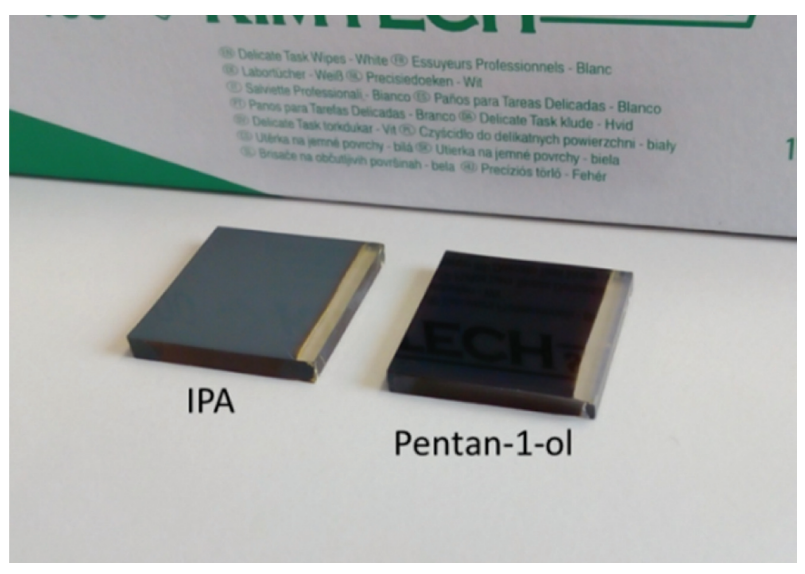

Figure 3: Digital photograph of $\mathrm{MAPbl}_{3}$ layers prepared from IPA and pentan-1-ol solvents.

the low permittivity of the conversion solution may reduce the intensity of Ostwald ripening and gives rise to a softer crystal growth of the perovskite phase in the absorber film.

To assess the impact of the low permittivity of pentan-1-ol on the crystallinity and conversion of $\mathrm{MAPbI}_{3}$ films, XRD is employed and compared to layers prepared from pristine IPA (Fig. 4). From both solvents, the three strongest Bragg reflections are observed at $2 \theta$ angles of $14.1^{\circ}, 28.3^{\circ}$, and $31.8^{\circ}$, which can be assigned to the (110), (220), and (310) planes, respectively, confirming the tetragonal phase of $\mathrm{MAPbI}_{3}$ in the $I 4 / \mathrm{mcm}$ symmetry [44]. Highly crystalline perovskite grains are obtained in all cases. The reflex at $12.67^{\circ}$ indicates unreacted $\mathrm{PbI}_{2}$ and can be assigned to the (001) lattice planes of the $2 \mathrm{H}$ hexagonal phase of $\mathrm{PbI}_{2}$ (ICSD file no. 98-002-4262, space group: $P-3 m 1$ [no. 164]). Changing the solvent from

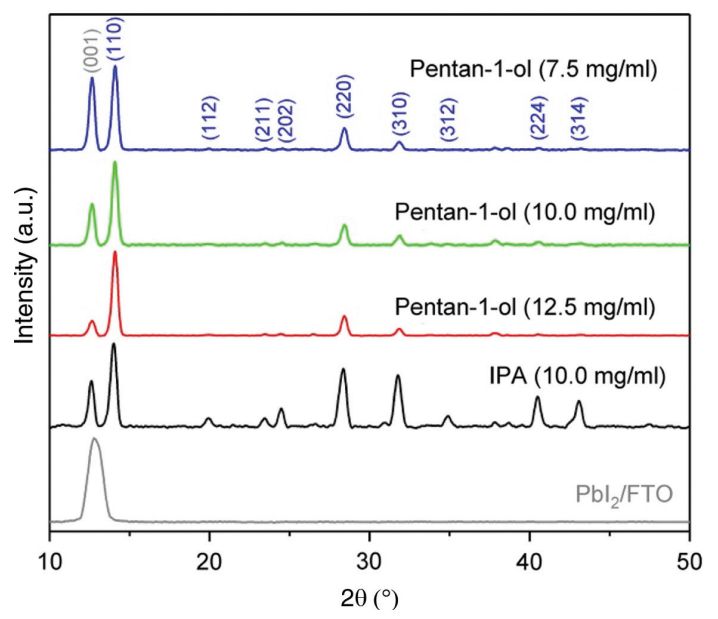

Figure 4: $\mathrm{X}$-ray diffraction patterns of the $\mathrm{Pbl}_{2}$ precursor layer and $\mathrm{MAPbl}_{3}$ films prepared by the sequential deposition process in different alcoholic MAl solutions. The gray and purple labels correspond to reflexes from the $\mathrm{Pbl}_{2}$ and $\mathrm{MAPbl}_{3}$ phase, respectively.
IPA to pentan-1-ol and keeping the concentration of MAI constant at $10.0 \mathrm{mg} / \mathrm{mL}$ lead to similar reflex intensities for the residual $\mathrm{PbI}_{2}$. However, by simply increasing the concentration of the MAI/pentan-1-ol solution (i.e. up to $12.5 \mathrm{mg} / \mathrm{mL}$ ), the reflex intensity at $12.67^{\circ}$ can be significantly lowered. Furthermore, the use of pentan-1-ol allows us to completely avoid unfavourable $\mathrm{PbI}_{2}$ by prolonging the immersion time to approximately $6 \mathrm{~min}$, whereby the high-quality and compact surface properties are not altered. This observation confirms that the dissolutionrecrystallisation phenomenon in low-permittivity pentan1-ol is strongly dependent on the concentration of MAI. Wang et al. [45] have investigated the influence of unconverted $\mathrm{PbI}_{2}$ in two-step deposited $\mathrm{MAPbI}_{3}$ layers and have observed that excess quantities of approximately $5 \%$ result in higher trap-state densities, increased excitonbinding energies, and faster carrier recombination. All of these characteristics result in adverse effects for the photovoltaic performance [45]. Cao et al. [46], on the other hand, have revealed that trace amounts of $\mathrm{PbI}_{2}$ may have beneficial effects on the device performance. By acting as a blocking layer between the compact $\mathrm{TiO}_{2}$ and the perovskite layer, an optimum residual quantity of roughly $2 \%$ is stated to yield the highest photovoltaic efficiencies. Considering both of these findings, the facile control of the $\mathrm{PbI}_{2}$ conversion ratio accompanied by the negligible change in surface characteristics with the aid of pentan1-ol may lead to enhanced charge carrier transport and optoelectronic properties.

Steady-state and transient PL measurements were performed on the as-prepared layers to investigate the charge carrier recombination dynamics. Generally, trap states have undesirable impacts on the solar cell performance, and although these have been periodically reported to be rather shallow in hybrid perovskite materials $(\sim 0.01-0.13 \mathrm{eV})[47,48]$, it is accepted that high photovoltaic efficiencies can only be achieved with less trap states [49]. The peak emission wavelength of $\mathrm{MAPbI}_{3}$ films prepared from pristine IPA is located at $790 \mathrm{~nm}$, whereas by using pentan-1-ol in the conversion step, a slight blue shift to $788 \mathrm{~nm}$ is observed (Fig. 5a). The latter can be attributed to the passivation of trap states near the band edges, which results in a slight increase of the band gap $\left(E_{\text {gap }}\right)$ [50]. The transient PL technique yields relevant information about the lifetimes of excited carriers in the photoactive layer and is indirectly giving a measure of the charge carrier collection efficiency. In that sense, the relaxation time is proportional to the carrier lifetime, representing a direct indicator for radiative or nonradiative recombination caused by impurities and/or crystal defects [51]. As $\mathrm{MAPbI}_{3}$ exhibits low exciton-binding energies, about 

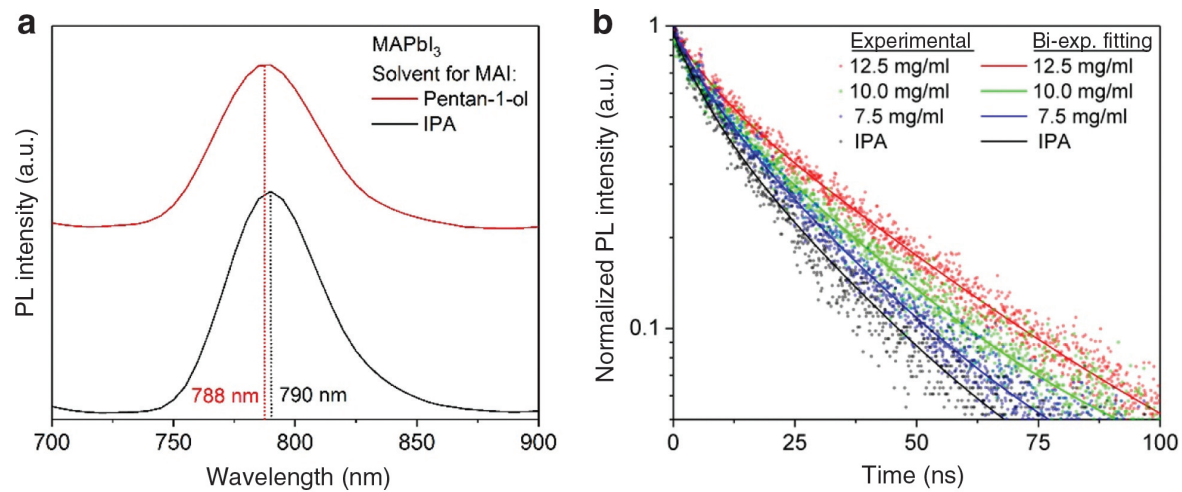

Figure 5: (a) Steady-state photoluminescence spectra of sequentially deposited $\mathrm{MAPbl}_{3}$ films on pure glass/FTO substrates prepared with IPA and pentan-1-ol as the solvent in the conversion reaction with a MAl concentration of $10 \mathrm{mg} / \mathrm{mL}$, respectively. (b) Nanosecond timeresolved photoluminescence decays of $\mathrm{MAPbl}_{3}$ films prepared from IPA and pentan-1-ol. The MAl concentrations are denoted in the graph. The PL decay time constants are obtained by a biexponential fitting procedure.

Table 1: List of the fitting parameters for the biexponential fits in Figure $5 \mathrm{~b}$.

\begin{tabular}{lrrrrr}
\hline Solution & A1 & A2 & t1 [ns] & t2 [ns] & t ${ }_{\text {average [ns] }}$ \\
\hline IPA, $10.0 \mathrm{mg} / \mathrm{mL}$ & $0.35 \pm 0.01$ & $0.58 \pm 0.01$ & $5.99 \pm 0.29$ & $23.85 \pm 0.41$ & $15.83 \pm 0.34$ \\
Pentan-1-ol, $7.5 \mathrm{mg} / \mathrm{mL}$ & $0.29 \pm 0.01$ & $0.65 \pm 0.01$ & $6.87 \pm 0.35$ & $25.10 \pm 0.36$ & $18.22 \pm 0.35$ \\
Pentan-1-ol, $10.0 \mathrm{mg} / \mathrm{mL}$ & $0.21 \pm 0.01$ & $0.67 \pm 0.01$ & $5.44 \pm 0.35$ & $27.65 \pm 0.30$ & $19.57 \pm 0.28$ \\
Pentan-1-ol, $12.5 \mathrm{mg} / \mathrm{mL}$ & $0.26 \pm 0.01$ & $0.68 \pm 0.01$ & $7.22 \pm 0.34$ & $34.38 \pm 0.38$ & $25.25 \pm 0.35$ \\
\hline
\end{tabular}

half of the excitons are self-dissociated at room temperature after photoexcitation [52]. Therefore, we expect that for the excitation wavelength and intensity used in the measurements the PL signal arises mostly from radiative recombination of free charges. No selective electron or hole contacts were added, and quenching effects on photoexcited species can be excluded. As demonstrated in Figure 5b, perovskite layers from MAI/pentan-1-ol solutions generally reveal slower PL decay times compared to films prepared from IPA. Individual fits of the four

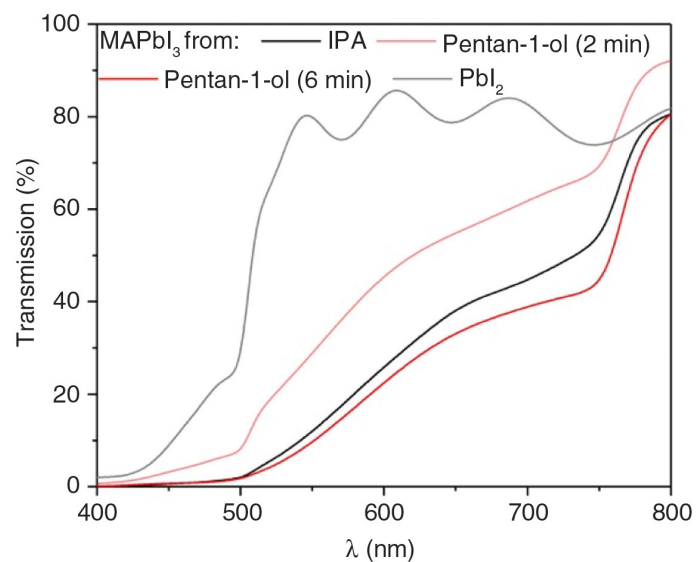

Figure 6: Ultraviolet-visible transmission spectra of the $\mathrm{Pbl}_{2}$ precursor layer and $\mathrm{MAPb}_{3}$ films prepared at room temperature from MAI solutions in IPA (10.0 mg/mL) and pentan-1-ol (12.5 mg/mL). The immersion time in the MAI/IPA solution is $2 \mathrm{~min}$. transients are provided in the Supporting information as Fig. S2. The decays are not monoexponential, indicating the presence of at least different kinds of impurities and/or defects. As a reliable assignment of the decays to different kinds of defect sites was not possible, average recombination lifetimes were determined by weighting the lifetimes from $\mathrm{t} 1$ and $\mathrm{t} 2$ with their amplitudes A1 and A2 (Tab. 1). The resulting average charge carrier lifetimes increase constantly with increasing concentrations of MAI/pentan1-ol, revealing values of $18.22,19.57$, and 25.25 ns for 7.5 ,

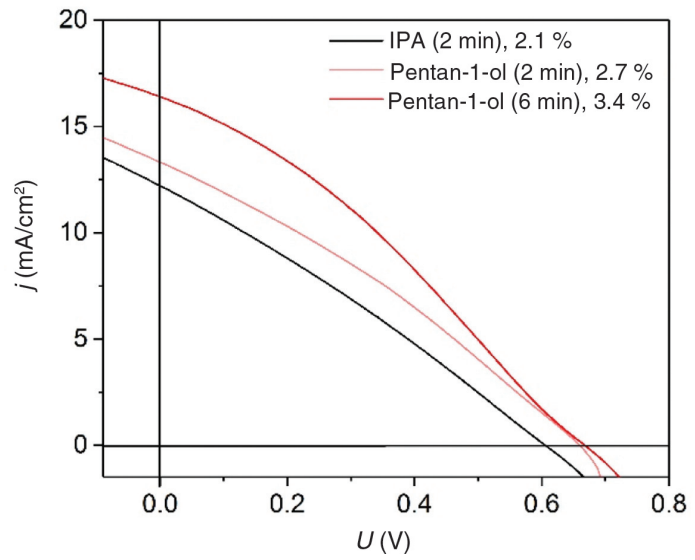

Figure 7: J-V measurements of planar $\mathrm{MAPbl}_{3}$-based devices prepared sequentially from pristine IPA and pentan-1-ol solutions (see Experimental Section for preparation and characterisation details). 

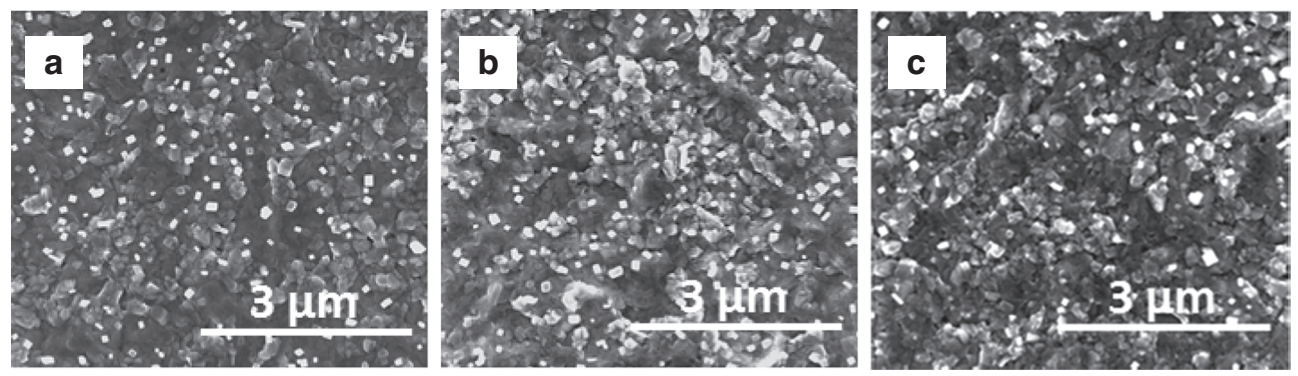

Figure 8: Top-view SEM images of $\mathrm{MAPbl}_{3}$ absorber layers prepared from MAl/iso-amyl alcohol solutions with concentrations of (a) 15.0, (b) 12.5 , and (c) $10.0 \mathrm{mg} / \mathrm{mL}$, respectively. Dipping was performed at room temperature for $2 \mathrm{~min}$.

10.0, and $12.5 \mathrm{mg} / \mathrm{mL}$, respectively. $\mathrm{MAPbI}_{3}$ films prepared from pristine IPA demonstrate the shortest average PL lifetime with an average value of 15.83 ns. The decay time constants are obtained by a biexponential fitting procedure. Probably the increase of charge carrier lifetimes is mainly associated to less trap states due to the increased surface coverage and improved compactness of the films.

Ultraviolet-visible transmission spectroscopy was used to investigate the effects of the low permittivity of pentan-1-ol on the optical properties of $\mathrm{MAPbI}_{3}$ films (Fig. 6). For all investigated samples, the onset of absorption remains relatively unaffected at about $790 \mathrm{~nm}$, corresponding to an $E_{\text {gap }}$ of approximately $1.57 \mathrm{eV}$. This is comparable to reported values for $\mathrm{MAPbI}_{3}$ films prepared by the two-step method [38-40, 53]. The strength of absorption in the visible range for films prepared from pentan-1-ol can be significantly increased via prolonging the immersion time from 2 to $6 \mathrm{~min}$. This results in an improved conversion ratio for $\mathrm{PbI}_{2}$ while simultaneously avoiding a modification of the surface morphology. The inferior absorbance of the conventionally prepared perovskite device is ascribed to the reduced surface coverage and increased number of pinholes, both of which lead to a considerable leakage of light.

Figure 7 shows the $J-V$ curves of planar perovskite solar cells prepared either from IPA or from pentan-1ol. Although cell preparation is far from being optimised and the overall performance of the cells is very low (it is expected that high charge transfer resistances of the nonoptimised $\mathrm{TiO}_{2}$ layer lead to low current densities), the effects of the improved surface morphology by permittivity adjustment can nevertheless be demonstrated, as all other parameters were strongly kept constant. The conventionally prepared reference cell from IPA shows an efficiency of about $2.1 \%$. The efficiency is improved to $2.7 \%$ by using the lower permittivity solvent pentan-1-ol and immersion for $2 \mathrm{~min}$; by prolonging the immersion time to $6 \mathrm{~min}$, the efficiency is even further improved to $3.4 \%$. This improvement by more than $50 \%$ can be attributed to the better conversion ratio as well as to the resulting stronger optical absorption. The results indicate that the control of the permittivity of the MAI solvent can positively influence the perovskite crystallisation behaviour and film properties, with the main goals to achieve high surface coverages and minor amounts of residual $\mathrm{PbI}_{2}$.

The influence of a second alcohol on the surface properties is investigated in order to confirm the correlation between the permittivity and intensity of dissolutionrecrystallisation. Iso-amyl alcohol (3-methyl-1-butanol, $\geq 98.5 \%$; Carl Roth, Karlsruhe, Germany) also exhibits a lower permittivity (15.2 As/Vm) compared to IPA associated with a high dipole moment $(1.8 \mathrm{D})$ [36]. Top-view SEM images show that the perovskite crystal growth is similarly altered, resulting in smooth and dense absorber layers (Fig. 8). Due to the relatively large dipole moment of isoamyl alcohol, a highest MAI concentration of $15.0 \mathrm{mg} / \mathrm{mL}$ can be obtained. The latter yields the most compact perovskite layers, whereas lower concentrations result in slight irregularities of the top surface. Both findings indicate an equal behaviour in the crystallisation process related to pentan-1-ol. The basic interactions between the permittivity and the associated nucleation kinetics will be an important parameter for future research on the sequential deposition of perovskite solar cells.

\section{Conclusions}

In summary, pentan-1-ol is used as alternative solvent for the dissolution of MAI in the sequential deposition of planar perovskite absorber layers. The dissolutionrecrystallisation behaviour, typically associated with the IPA solvent, can be successfully inhibited due to the lower permittivity, and an in situ intercalation is expected to occur. As a consequence, the obtained surfaces are considerably dense as well as compact and resemble in morphology those layers prepared by vapour-assisted techniques. The highly crystalline films demonstrate 
enhanced charge carrier lifetimes and strong optical absorption. Furthermore, the amount of unreacted $\mathrm{PbI}_{2}$ can be easily controlled by the conversion reaction time without significantly alternating the high-quality surface morphology. Planar devices prepared from pentan-1-ol demonstrate an increase in the PCE of up to $\sim 60 \%$. Thus, it is assumed that a control of the solvent permittivity provides a favourable way for the fabrication of planar perovskite solar cells from the native two-step synthesis.

\section{Highlights}

1. A modified sequential deposition for planar perovskite films is suggested.

2. The solvent permittivity determines the intensity of Ostwald ripening.

3. The absorber surface quality and compactness are significantly improved by using lower-permittivity pentan-1-ol.

4. Charge carrier lifetimes and optoelectronic properties are enhanced.

5. The photovoltaic performance is significantly improved with compact layers.

Acknowledgements: We acknowledge the support for PL measurements by Dr. Stephan Heise from the Energy and Semiconductor Research Group, Department of Physics, Carl von Ossietzky University of Oldenburg, Germany.

\section{References}

[1] J. H. Im, C. R. Lee, J. W. Lee, S. W. Park, and N.G. Park, Nanoscale 3, 4088 (2011).

[2] H. S. Kim, C. R. Lee, J. H. Im, K. B. Lee, T. Moehl, et al., Sci. Rep. 2, 591 (2012).

[3] M. M. Lee, J. Teuscher, T. Miyasaka, T. N. Murakami, and H. J. Snaith, Science 338, 643 (2012).

[4] S. D. Stranks, G. E. Eperon, G. Grancini, C. Menelaou, M. J. P. Alcocer, et al., Science 342, 341 (2013).

[5] J. M. Ball, M. M. Lee, A. Hey, and H. J. Snaith, Energy Environ. Sci. 6, 1739 (2013).

[6] M. Z. Liu, M. B. Johnston, and H. J. Snaith, Nature 501, 395 (2013).

[7] H. P. Zhou, Q. Chen, G. Li, S. Luo, T. B. Song, et al., Science 345, 542 (2014).

[8] A. Kojima, K. Teshima, Y. Shirai, and T. Miyasaka, J. Am. Chem. Soc. 131, 6050 (2009).

[9] Renewable Energy Laboratory (NREL). https://www.nrel.gov/ $\mathrm{pv} /$ assets/pdfs/best-research-cell-efficiencies-190416.pdf (accessed 08.06.2019).

[10] M. A. Green, K. Emery, Y. Hishikawa, W. Warta, and E. D. Dunlop, Prog. Photovoltaics 22, 701 (2014).
[11] C. Battaglia, A. Cuevas, and S. De Wolf, Energy Environ. Sci. 9, 1552 (2016).

[12] A. Miyata, A. Mitioglu, P. Plochocka, O. Portugall, J. T. W. Wang, et al., Nat. Phys. 11, 582 (2015).

[13] S. de Wolf, J. Holovsky, S. J. Moon, P. Loeper, B. Niesen, et al., J. Phys. Chem. Lett. 5, 1035 (2014).

[14] G. Giorgi, J. I. Fujisawa, H. Segawa, and K. Yamashita, J. Phys. Chem. Lett. 4, 4213 (2013).

[15] V. Fthenakis, Renew. Sust. Energy Rev. 13, 2746 (2009).

[16] W. Ke and M. G. Kanatzidis, Nat. Commun. 10, 965 (2019).

[17] D. Wang, M. Wright, N. K. Elumalai, and A. Uddin, Sol. Energ. Mat. Sol. 147, 255 (2016).

[18] D. Y. Son, J. H. Im, H. S. Kim, and N. G. Park, J. Phys. Chem. C 118, 16567 (2014).

[19] Y. Liu, M. Bag, L. A. Renna, Z. A. Page, P. Kim, et al., Adv. Energy Mater. 6, 1501606 (2016).

[20] J. Liu, G. Wang, K. Luo, Q. Ye, C. Liao, et al., Phys. Chem. Chem. Phys. 18, 617 (2017).

[21] M. C. Jung, S. R. Raga, L. K. Ono, and Y. B. Qi, Sci. Rep.-UK 5, 9863 (2015).

[22] Q. L. Wu, C. Xue, Y. Li, P. C. Zhou, W. F. Liu, et al., ACS Appl. Mater. Interf. 7, 28466 (2015).

[23] F. Hanusch, M. Petrus, and P. Docampo, in: Unconventional Thin Film Photovoltaics (Eds. E. Da Como, F. De Angelis, H. Snaith, A. Walke), The Royal Society of Chemistry, Cambridge, UK 2016, p. 32.

[24] T. B. Song, Q. Chen, H. P. Zhou, C. Y. Jiang, H. H. Wang, et al., J. Mater. Chem. A 3, 9032 (2015).

[25] Y. N. Chen, M. H. He, J. J. Peng, Y. Sun, and Z. Q. Liang, Adv. Sci. 3, 1500392 (2016).

[26] G. E. Eperon, V. M. Burlakov, P. Docampo, A. Goriely, and H. J. Snaith, Adv. Funct. Mater. 24, 151 (2014).

[27] K. Fu, C. T. Nelson, M. C. Scott, A. Minor, N. Mathews, et al., Nanoscale 8, 4181 (2016).

[28] D. Y. Liu and T. L. Kelly, Nat. Photonics 8, 133 (2014).

[29] J. Burschka, N. Pellet, S. J. Moon, R. Humphry-Baker, P. Gao, et al., Nature 499, 316 (2013).

[30] S. Yang, Y. Chen, Y. C. Zheng, X. Chen, Y. Hou, et al., RSC Adv. 5, 69502 (2015).

[31] T. Y. Hsieh, C. K. Huang, T. S. Su, C. Y. Hong, and T. C. Wei, ACS Appl. Mater. Interf. 9, 8623 (2017).

[32] Q. Chen, H. P. Zhou, Z. R. Hong, S. Luo, H. S. Duan, et al., J. Am. Chem. Soc. 136, 622 (2014).

[33] W. Zeng, X. Liu, X. Guo, Q. Niu, J. Yi, et al., Molecules 22, 520 (2017).

[34] J. H. Im, H. S. Kim, and N. G. Park, APL Mater. 2, 081510 (2014).

[35] C. Wohlfahrt, Static Dielectric Constants of Pure Liquids and Binary Liquid Mixtures, Springer, Berlin, Germany 1991.

[36] I. N. Smallwood, Handbook of Organic Solvent Properties, Arnold, London, UK 1996.

[37] M. Becker and M. Wark, Org. Electron. 50, 87 (2017).

[38] P. Docampo, F. C. Hanusch, S. D. Stranks, M. Doblinger, J. M. Feckl, et al., Adv. Energy Mater. 4, 1400355 (2014).

[39] P. Docampo, F. C. Hanusch, N. Giesbrecht, P. Angloher, A. Ivanova, et al., APL Mater. 2, 081508 (2014).

[40] B. E. Cohen, S. Gamliel, and L. Etgara, APL Mater. 2, 081502 (2014).

[41] J. H. Im, I. H. Jang, N. Pellet, M. Graetzel, and N. G. Park, Nature Nanotechnol. 9, 927 (2014). 
[42] Y. Z. Wu, A. Islam, X. D. Yang, C. J. Qin, J. Liu, et al., Energy Environ. Sci. 7, 2934 (2014).

[43] T. Y. Zhang, M. J. Yang, Y. X. Zhao, and K. Zhu, Nano Lett. 15, 3959 (2015).

[44] T. Baikie, Y. N. Fang, J. M. Kadro, M. Schreyer, F. X. Wei, et al., J. Mater. Chem. A 1, 5628 (2013).

[45] H. Y. Wang, M. Y. Hao, J. Han, M. Yu, Y. Qin, et al., Chem. Eur. J. 23, 3986 (2017).

[46] D. Y. H. Cao, C. C. Stoumpos, C. D. Malliakas, M. J. Katz, O. K. Farha, et al., APL Mater. 2, 091101 (2014).

[47] H. Oga, A. Saeki, Y. Ogomi, S. Hayase, and S. Seki, J. Am. Chem. Soc. 136, 13818 (2014).

[48] G. Gordillo, C. A. Otalora, and A. A. Ramirez, Phys. Chem. Chem. Phys. 18, 32862 (2016).
[49] N. K. Noel, A. Abate, S. D. Stranks, E. S. Parrott, V. M. Burlakov, et al., ACS Nano 8, 9815 (2014).

[50] Y. H. Shao, Z. G. Xiao, C. Bi, Y. B. Yuan, and J. S. Huang, Nature Commun. 5, 5784 (2014).

[51] K. F. Lin, S. H. Chang, K. H. Wang, H. M. Cheng, K. Y. Chiu, et al., Sol. Energy Mat. Sol. C 141, 309 (2015).

[52] T. C. Sum and N. Mathews, Energy Environ. Sci. 7, 2518 (2014).

[53] Z. Xiao, C. Bi, Y. Shao, Q. Dong, Q. Wang, et al., Energy Environ. Sci. 7, 2619 (2015).

Supplementary Material: The online version of this article offers supplementary material (https://doi.org/10.1515/zna-2019-0141). 